\title{
AN EXPLORATION OF PERSONALITY EFFECTS IN RISK DECISION MAKING
}

\author{
John Garvey \\ University of Limerick \\ and \\ Laura Lee \\ University of Limerick
}

\begin{abstract}
The notion of risk aversion provides an important starting point for mainstream theories relating to portfolio management and insurance purchasing. The current study uses prospect theory as a starting point for examining the role of personality effects, specifically risk propensity, in decision making. This personality construct emerges as an influencing factor on decisions when individuals are faced with potential gains. Conversely, the expectation of potential losses in a decision-making scenario reduces the influence of risk propensity in decision making.
\end{abstract}

\section{INTRODUCTION}

Conventional financial theory, as it relates to asset pricing and market efficiency, is concerned with aggregate responses to new information. Key theoretical developments such as modern portfolio theory and the capital asset pricing model (CAPM) rely on the assumption of a risk-averse investor who has the facility to rank assets according to a clearly defined risk-return tradeoff. As a result, individual personality differences have been largely ignored in mainstream financial theory. Over the past twenty years the effects of globalisation, improved technology and increasing leverage means that individual decisions and attitudes to risk can have major ripple effects. This is evidenced by several notable loss events that were initiated or aggravated by the characteristics of individual actors. ${ }^{1}$ These events subsequently eliminated or crippled organisations and in some cases produced significant market volatility. 
The role of personality on risk decision making has been neglected in favour of the assumption that individuals exhibit a diminishing marginal utility associated with wealth. This changed somewhat when the doctrine of traditional utility theory was challenged in the experimental work carried out by Kahneman and Tversky $(1974,1979,1981)$. They demonstrated that individuals very often preferred probable losses to certain losses. This behaviour was observed even though the expected magnitude of these losses exceeded certain losses. Individuals thus revealed a tendency to be risk seeking when presented with choices that would lead to losses. This experimental work clearly demonstrates the deficiencies of utility theory as a descriptive model of decision making under risk and encouraged further exploration of apparent violations of this traditional framework (Kahneman, Knetsch and Thaler, 1990; Rabin, 2000; Rabin and Thaler, 2001). Kahnemann and Tversky (1979) show that individuals exhibit 'the reflection effect', that is, they respond differently to the same problem depending on how it is framed.

An implicit assumption of prospect theory is that it is the positive or negative prospect that is presented to individuals that defines their subsequent choices. Individual differences in the form of personality characteristics are not considered as factors in the decision-making process. Furnham (1992) recognises that personality has different meanings that are dependent on the user's background or training. Psychologists take personality to mean the 'structure and dynamic inner processes' that individuals undertake as well as the 'stylistic consistencies in social behaviour' that emerge from an inner process (Furnham, 1992, p. 15). Within organisational behaviour research, Weiss and Adler (1984) note that variables such as self-esteem, self-actualisation and the fear of failure are identifiable personality factors on which theories of job motivation and performance rely. The use of psychometrics centres on the concept of trait theory, which proposes that people will usually behave in a certain manner dependant on their personality characteristics. According to trait theory, people behave consistently across situations and therefore it is possible to measure individuals' personality traits in myriad circumstances (Herriott, 1989). Social psychologists, such as Mischel (1968) and Schoemaker (1990), argue against this approach and propose that it is the situation that determines an individual's behaviour and not their personality. Slovic (1972) hypothesised that risk-taking propensity is discerned from a person's previous experiences, while Cho and Lee suggest that it be viewed as a 'behavioural tendency rather than a pure personality trait' (2006, p. 114) .

By applying psychometric analysis, this study updates prospect theory to show that inherent traits influence decision making and these traits become dominant in certain external conditions. We show that when the prospect of positive outcomes is available, risk-taking behaviour becomes dominant. This supports empirical evidence for existing behavioural biases that contribute to asset price 'bubbles' as well as potentially risky strategies within credit rating agencies and investment banks regarding credit risk. 


\section{METHODOLOGY AND RESULTS}

In this paper, we adopt the experimental framework used by Kahneman and Tversky (1981), which presents individuals with economic outcomes and associated probabilities, framed in positive and negative contexts. Participants in our study also completed a psychometric test that evaluates their self-attributed risk propensity. The probability questions will provide an indication of the individual's risk propensity in an actual decision-making process and the results are overlaid with the responses to the psychometric questionnaire. This approach elicits the individual's self-reported risk preferences and allows comparison against their actual behaviour. The data reported were collected from a sample of 521 participants in an online simulated trading game. ${ }^{2}$

For the probability based questions a total of five problems were used. Three problems were positively framed and two were negatively framed. An example of a positively framed problem was the following:

\section{Positive Prospect}

For the following set of problems, please select which option you would prefer to take:

Take a bet that pays $\$ 4,00080$ per cent of the time and $\$ 020$ per cent of the time?

Or

Take a bet that pays $\$ 3,000$ all the time?

Responses to a positively framed problem of this type provide an indication of the utility decisions of individuals when faced with potentially positive outcomes. The notion of diminishing marginal utility associated with wealth would predict that individuals are risk aversion and will select the certain $\$ 3,000$ in all cases. The negatively framed probability questions explore decisions relating to loss aversion as defined by Kahnemann and Tversky (1981). An example of a negatively framed prospect is captured as follows:

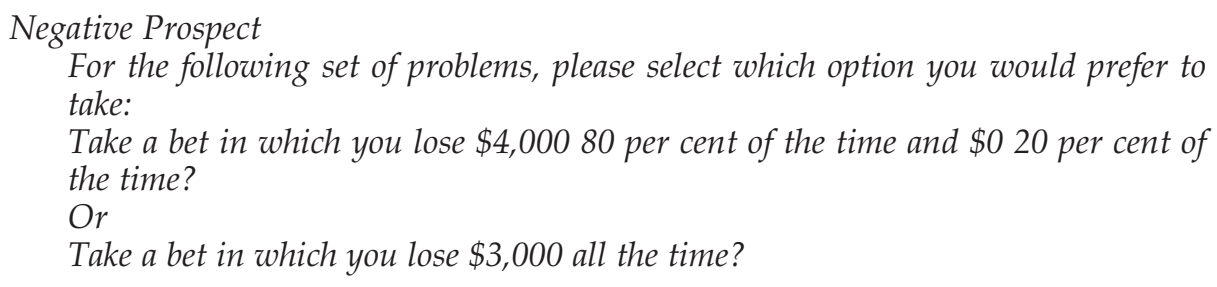

The responses to all five prospect questions are reported in Table 1. We can see that the framing of the problem had a significant impact on the subjects' preferences. For all positive prospect problems, a substantial majority of respondents favoured the certain or more probable response. For Problem 156 per cent preferred the certain outcome, compared to a 44 per cent preference for the uncertain outcome. This compares to Kahnemann and Tversky's (1979) results of 80 per cent 
Garvey \& Lee

TABLE I: RESPONSE TO POSITIVE AND NEGATIVE PROSPECTS

\begin{tabular}{|c|c|c|c|}
\hline \multicolumn{2}{|c|}{ Positive Prospects } & \multicolumn{2}{|c|}{ Negative Prospects } \\
\hline Problem I: & $(£ 4,000,0.80)<(£ 3,000)^{\dagger}$ & Problem 2: & $(-£ 4,000,0.80)>(-£ 3,000)$ \\
\hline$\%$ Response & {$[56 ; 80]^{\ddagger}$} & $\%$ Response & {$[65 ; 92]$} \\
\hline Problem 3: & $\begin{array}{l}(£ 3,000,0.90)> \\
(£ 6,000,0.45)\end{array}$ & Problem 4: & $(-£ 3,000,0.90)<(-£ 6,000,0.45)$ \\
\hline$\%$ Response & {$[72 ; 86][28 ; 14]$} & $\%$ Response & {$[68 ; 92]$} \\
\hline Problem 5: & $\begin{array}{l}(£ 3,000,0.0002)< \\
(£ 6,000,0.0001)\end{array}$ & & \\
\hline$\%$ Response & {$[35 ; 27][65 ; 73]$} & & \\
\hline
\end{tabular}

${ }^{\dagger}$ This shows the responses in favour of the certain option $(£ 3,000)$ over the uncertain option $(80$ per cent probability of winning $£ 4,000)$.

‡Responses are summarised in percentage form. The first number in each bracket represents the results found in this study. The numbers in bold represent Kahneman and Tversky's (1979) original findings.

and 20 per cent respectively. Responses to Problems 2, 3, 4 and 5 match Kahnemann and Tversky's (1979) findings more closely. That is, participants demonstrate risk aversion in the positive domain accompanied by risk seeking in the negative domain. Kahneman (2003, p. 702) suggests that although there is no substantial difference between the versions (positive or negative), 'they evidently evoke different associations and evaluations'. This reference to the inner processes of individual actions or behaviour recognises that there are individual differences. However, prospect theory relegates the influence of personality factors in decision making in favour of environment. McMartin (1995, p. 5) defines personality 'as a developing system of those distinctive emotional, cognitive and spiritual attributes that manifest themselves in the individual's characteristic behaviour at any point in the life course'. This interpretation of personality recognises that people's judgements and choices are not static but are developing and evolving through and in response to changing external conditions. The role of personality in decision making thus presents an ideal avenue to explore and extend the results originally proposed in prospect theory, which presented a more static and aggregated view of decision making.

Recognising that personality is an evolving, living process, participants in our study completed a psychometric test that consisted of a series of thirty-one questions. The test assessed five different personality constructs, namely, Risk Taking, Risk Avoidance, Education, Math Ability and Competitiveness. ${ }^{3}$ The two relevant constructs, risk taking and risk avoidance, were interspersed amongst the other constructs and both scales had ten associated questions. Table 2 summarises the descriptive statistics for risk taking and risk aversion scores.

Respondents were asked to consider a statement like 'Seek adventure' or 'Am willing to try anything once'. They were then required to assess, on a Likert-type scale, the appropriateness of this statement as a description of their own personality. Table 3 details the questions relating to risk propensity. The questions relating to risk taking/risk aversion personality constructs were drawn from the Jackson Personality Inventory and Tellegen's Multidimensional Personality 
An Exploration of Personality Effects in Risk Decision Making

TABLE 2: DESCRIPTIVE STATISTICS FOR RISK AVERSION AND RISK TAKING

\begin{tabular}{lccccc}
\hline & N & Minimum & Maximum & Mean & Standard Deviation \\
\hline Risk Aversion & 521 & 10 & 46 & 26.34 & 6.883 \\
Risk Taking & 521 & 14 & 48 & 34.14 & 6.188 \\
\hline
\end{tabular}

Questionnaire (MPQ). The response to the two risk-related personality constructs, risk taking and risk aversion, are significantly negatively correlated $(\mathrm{p}=-0.95)$. The strong inverse relationship demonstrates the consistency of the responses produced by the questionnaire. Individual results on self-attributed risk propensity were overlaid on the 'prospect-style' risk choices that were separately selected by participants. Participants' scores for risk taking and risk aversion are respectively categorised into low, medium and high levels. For risk aversion, low scores $(\mathrm{N}=$ 162) ranged $10-22$, medium scores $(\mathrm{N}=166) 23-28$ and high scores $(\mathrm{N}=193)$ 29-46. For risk taking, low scores $(\mathrm{N}=181)$ ranged $14-31$, medium scores $(\mathrm{N}=174)$ 32-37 and high scores $(\mathrm{N}=166)$ 38-48. This method of sub-grouping was decided upon so as to split the groups as evenly as possible.

Furthermore, the actual risk choices undertaken by respondents are categorised as consistent with prospect theory and are labelled 'For'. Conversely, responses that contradict prospect theory are categorised as 'Against'. The results for Problem 1 are summarised in Table 3. Respondents identified as high-level risk takers exhibit low risk aversion in economic decisions that are positively framed. This result is statistically significant $\left(X^{2}=12.590 ; p<0.01\right)$ and it supports the notion of personality effects emerging under specific conditions. In the positive domain, those cautious participants in our sample more closely adhere to the risk-averse individual described in previous studies. Participants who score highly on the risk aversion construct will prefer the certain option in a positive prospect situation.

TABLE 3: A CROSS-TABULATION OF PROBLEM I, A POSITIVE PROSPECT PROBLEM, WITH RISK TAKING

\begin{tabular}{|c|c|c|c|c|c|c|}
\hline \multirow{2}{*}{\multicolumn{3}{|c|}{ Positive Prospect Problem }} & \multicolumn{4}{|c|}{ Risk-Taking Propensity } \\
\hline & & & Low & Medium & High & Total \\
\hline \multirow[t]{4}{*}{ Prob_I } & Against & Count & 61 & 82 & 86 & 229 \\
\hline & & \% within RT & $33.7 \%$ & $47.1 \%$ & $51.8 \%$ & $44.0 \%$ \\
\hline & For & Count & 120 & 92 & 80 & 292 \\
\hline & & $\%$ within RT & $66.3 \%$ & $52.9 \%$ & $48.2 \%$ & $56.0 \%$ \\
\hline \multirow[t]{2}{*}{ Total } & & Count & $|8|$ & 174 & 166 & 521 \\
\hline & & \% within RT & $100.0 \%$ & $100.0 \%$ & $100.0 \%$ & $100.0 \%$ \\
\hline
\end{tabular}

* The value of Pearson's chi-square for this cross-tabulation is 12.590 , with a 2-tailed significance level of $p=$ 0.002 .

** The weighting for this problem is 56 per cent For the original findings and 44 per cent Against.

*** '\% within RT' means for those participants who ranked their risk-taking propensity (RT) the percentage of those whose responses are consistent (For)/not consistent (Against) with prospect theory. 
Garvey \& Lee

The results from Problem 1 are reinforced by the results produced in Problem 3. ${ }^{4}$ These findings are distinct from the generalised results provided by Kahnemann and Tversky $(1979,1981)$, who note that all individuals, irrespective of their risk preferences, demonstrate a tendency towards risk aversion in the positive domain. These findings show that individuals are influenced by their risk preferences, thus creating a more nuanced interpretation of decision-making behaviour than that put forward by prospect theory.

The influence of personality effects in decision making is inconclusive when individuals are presented with potentially negative outcomes. The results for Problem 2 are not statistically significant $\left(X^{2}=1.209 ; p=0.546\right)$, thus indicating that the response selection does not consistently increase or decrease in accordance with a greater level of risk taking. According to prospect theory, individuals will demonstrate a tendency to seek risk in the negative domain. This indicates that when faced with the choice of a certain or a probable outcome, individuals tend to assume greater risk and choose the probable option, which offers the higher return. The results, reported in Table 4 , indicate that this tendency will prevail irrespective of an individual's level of risk taking.

This is similarly evident in the risk aversion results, where the trend is also non-significant $\left(X^{2}=1.642 ; p=0.440\right)$. These results suggest that in the negative domain, an individual's personality is less of a factor in their decision making. This might imply that in the negative domain, situational factors are more prevalent than personality preferences. The results for Problem 4 are similar to the results for Problem 2. ${ }^{5}$ For Problems 1 and 3, the subjects' responses were significantly impacted by their risk preferences, evidence which supports a trait approach. For Problems 2 and 4, the results showed that instead of being influenced by their risk propensity, subjects showed a greater sensitivity to the presentation, thus supporting a state approach (one that identifies the external environment as the primary influence on decision making). The presence of loss aversion supports the results found for these problems, where it is considered that

TABLE 4: A CROSS-TABULATION OF PROBLEM 2,A NEGATIVE PROSPECT PROBLEM,WITH RISK TAKING

\begin{tabular}{|c|c|c|c|c|c|c|}
\hline \multirow{2}{*}{\multicolumn{3}{|c|}{ Negative Prospect Problem }} & \multicolumn{4}{|c|}{ Risk-Taking Propensity } \\
\hline & & & Low & Medium & High & Total \\
\hline \multirow[t]{4}{*}{ Prob_2 } & Against & Count & 68 & 56 & 56 & 180 \\
\hline & & \% within RT & $37.6 \%$ & $32.2 \%$ & $33.7 \%$ & $34.5 \%$ \\
\hline & For & Count & 113 & 118 & 110 & 341 \\
\hline & & \% within RT & $62.4 \%$ & $67.8 \%$ & $66.3 \%$ & $65.5 \%$ \\
\hline \multirow[t]{2}{*}{ Total } & & Count & $|8|$ & 174 & 166 & 521 \\
\hline & & \% within RT & $100.0 \%$ & $100.0 \%$ & $100.0 \%$ & $100.0 \%$ \\
\hline
\end{tabular}

* The value of Pearson's chi-square for this cross-tabulation is 1.209 , at a non-significant level of $p=0.546$.

** The weighting for this problem is $65 \%$ For the original findings and $34 \%$ Against.

*** '\% within RT' means for those participants who ranked their risk-taking propensity (RT) the percentage of those whose responses are consistent (For)/not consistent (Against) with prospect theory. 
losses 'loomed larger' and, as a result, caused greater interference with the subject's decision making process.

Problem 5 is framed as a positive prospect problem that is designed to elicit participants' ability to rank low probability outcomes. In this context we find little relationship between the choices undertaken by participants and their self-attributed risk propensity $\left(X^{2}=3.013 ; p=0.222\right)$. Kahneman and Tversky (1979) proposed that in the situation where individuals are presented with the possibility of winning, but where it is not very probable, then they will be likely to choose the prospect that offers the larger gain. This is similar to Problems 2 and 4 (where the larger gain is more preferable), and might explain why there is not a significant influence exerted by risk preferences.

\section{CONCLUSIONS}

The success of prospect theory in exploring behaviour under alternating conditions has encouraged a move away from a rigid adherence to the traditional theoretical assumption of a risk-averse economic actor. Prospect theory presents the external context as a key driver in risk decision making. It thus provides an unexpected springboard for an examination of individual differences or traits in decision making.

In this paper, prospect theory provides a framework for the exploration of self-attributed risk propensity. The focus of this paper is the internal processes of individuals when required to undertake a choice with alternating prospects. Although the paper concerns itself with subjective interpretations of risk, we propose that it is appropriate 'to talk of individuals without individualising' (Loffe, 1999 , p. 8). That is, our analysis of subjectivity is stable and can be extended beyond the sample population considered here.

The results shown in this paper support the notion that decision making is context-dependant, as demonstrated in prospect theory. However the new insight offered here is that it is not simply the framing of a problem that influences choice. Under specific conditions the inner processes of an individual are activated and become evident in the choices they make. We show that the positive framing of a problem allows high-level risk takers to be identified in their choices. Furthermore, personality effects become muted and difficult to observe in the choices made under negatively framed problems.

\section{NOTES}

1 Some of the well-documented loss events that fall within this category include the $\$ 7.2$ billion loss at Société Générale (Jerome Kerviel), the $£ 827$ million loss at Barings Bank (Nick Leeson), the £557 million loss at Resona Holdings (Toshihide Iguchi), the $\$ 2.6$ billion loss at Sumitomo Corporation (Yasuo Hamanaka), the $£ 350$ million loss at Allied Irish Banks (John Rusnak) and the AU\$360 million loss at National Australia Bank (Luke Duffy). 
2 Participants used in this study were registered traders on <www.bullbearings.co.uk>. Of the 521 sample, 459 were male with an average age of 27.93 years, while 62 were female with an average age of 29.03 years.

3 The psychometric questionnaire used in this research is validated by Goldberg et al. (2006) and is publicly available on <http://ipip.ori.org/>.

4 Problem 3 is also a positive prospect problem and the results are similarly found to be statistically significant $\left(X^{2}=6.535 ; p<0.05\right)$. The results for risk aversion also return a significant trend $\left(X^{2}=6.727 ; p=0.035\right)$. Participants' propensity to select For is observed to increase as their risk aversion score increases.

5 The results for Problem 4 (also a negatively framed prospect) are not significant $\left(X^{2}=0.482 ; p=0.786\right)$. There is thus no observable trend in risk choices being related to the risk taking personality construct. The results for the risk aversion construct are similarly not significant $\left(X^{2}=0.972 ; p=0.615\right)$.

\section{REFERENCES}

Cho, J. and Lee, J. (2006). An Integrated Model of Risk and Risk-Reducing Strategies, Journal of Business Research, Vol. 59, No. 1, pp. 112-120.

Furnham, A. (1992). Personality at Work: The Role of Individual Differences in the Workplace, New York, NY: Routledge.

Goldberg, L.R., Johnson, J.A., Eber, H.W., Hogan, R., Ashton, M.C., Cloninger, C.R. and Gough, H.C. (2006). The International Personality Item Pool and the Future of Public-Domain Personality Measures, Journal of Research in Personality, Vol. 40, No. 1, pp. 84-96.

Herriott, P. (1989). Assessment and Selection in Organisations: Methods and Practices for Research and Applications, Chichester: Wiley.

Kahneman, D. (2003). A Perspective on Judgment and Choice: Mapping Bounded Rationality, American Psychologist, Vol. 58, No. 9, pp. 697-720.

Kahneman, D., Knetsch, J.L. and Thaler, R.H. (1990). Experimental Tests of the Endowment Effect and the Coase Theorem, Journal of Political Economy, Vol. 98, No. 6, pp. 1325-1348.

Kahneman, D. and Tversky, A. (1974). Judgment under Uncertainty: Heuristics and Biases, Science, Vol. 185, No. 4157, pp. 1124-1131.

Kahneman, D. and Tversky, A. (1979). Prospect Theory: An Analysis of Decision under Risk, Econometrica, Vol. 47, No. 2, pp. 263-292.

Kahneman, D. and Tversky, A. (1981). The Framing of Decisions and the Psychology of Choice, Science, Vol. 211, No. 4481, pp. 453-458.

Loffe, H. (1999). Risk and 'The Other', Cambridge: Cambridge University Press.

McMartin, J. (1995). Personality Psychology: A Student-Centered Approach, London: Sage Publications.

Mischel, W. (1968). Personality and Assessment, New York, NY: Wiley.

Rabin, M. (2000). Risk Aversion and Expected-Utility Theory: A Calibration Theorem, Econometrica, Vol. 68, No. 5, pp. 1281-1292.

Rabin, M. and Thaler, R.H. (2001). Anomalies: Risk Aversion, Journal of Economic Perspectives, Vol. 15, No. 1, pp. 219-232.

Schoemaker, P.J.H. (1990). Are Risk-Attitudes Related across Domains and Response Modes?, Management Science, Vol. 36, No. 12, pp. 1451-1463. 
Slovic, P. (1972). Psychological Study of Human Judgment: Implications for Investment Decision Making, Journal of Finance, Vol. 27, No. 4, pp. 779-799.

Weiss, H. and Adler, S. (1984). Personality and Organizational Behavior, Research in Organizational Behavior, Vol. 4, pp. 711-718. 